A. Benkirane (Atlas Fès)

A. Elmahi (Fès)

D. Meskine (Atlas Fès)

\title{
AN EXISTENCE THEOREM FOR A CLASS OF ELLIPTIC PROBLEMS IN $L^{1}$
}

Abstract. We prove an existence result for solutions of some class of nonlinear elliptic problems having natural growth terms and $L^{1}$ data.

1. Introduction. Let $\Omega$ be an open bounded subset of $\mathbb{R}^{N}, N \geq 2$, with the segment property, and let $f \in L^{1}(\Omega)$. Consider the following nonlinear Dirichlet problem:

$$
A(u)+g(x, u, \nabla u)=f
$$

where $A(u)=-\operatorname{div}(a(x, u, \nabla u))$ is a Leray-Lions operator defined on $D(A)$ $\subset W_{0}^{1} L_{M}(\Omega)$, with $M$ an $N$-function which satisfies the $\Delta_{2}$-condition, and $g$ is a nonlinearity having "natural growth" and satisfies the classical "sign condition" with respect to $u$.

In the variational case (i.e. where $f \in W^{-1} E_{\bar{M}}(\Omega)$ ), it is well known that Gossez solved (1.1) in the case where $g$ depends only on $x$ and $u$. If $g$ does depend also on $\nabla u$, an existence theorem has recently been proved by Benkirane and Elmahi in [4] and [5].

In the case where $f \in L^{1}(\Omega)$, they also give an existence result in [6] if the nonlinearity $g$ satisfies further the following coercivity condition:

$$
|g(x, s, \zeta)| \geq \beta M(|\zeta| / \mu) \quad \text { for }|s| \geq \gamma .
$$

It is our purpose, in this paper, to prove an existence result for some class of problems of the kind (1.1), without assuming the coercivity condition (1.2). The technical method used here allows us not only to generalize the result of [21], but also to give a different proof for it.

2000 Mathematics Subject Classification: 35J25, 35J60.

Key words and phrases: strongly nonlinear problems in $L^{1}$, truncations, OrliczSobolev space. 
For the classical variational case in Orlicz spaces, the reader is referred, for example, to $[3-5,7,15-18]$, and for some results in the $L^{p}$ case, to $[2$, $10-14,20,21]$.

\section{Preliminaries}

2.1. Let $M: \mathbb{R}^{+} \rightarrow \mathbb{R}^{+}$be an $N$-function, i.e. $M$ is continuous, convex, with $M(t)>0$ for $t>0, M(t) / t \rightarrow 0$ as $t \rightarrow 0$ and $M(t) / t \rightarrow \infty$ as $t \rightarrow \infty$. Equivalently, $M$ admits a representation $M(t)=\int_{0}^{t} a(s) d s$ where $a: \mathbb{R}^{+} \rightarrow \mathbb{R}^{+}$is nondecreasing, right continuous, with $a(0)=0, a(t)>0$ for $t>0$ and $a(t)$ tends to $\infty$ as $t \rightarrow \infty$.

The $N$-function $\bar{M}$ conjugate to $M$ is defined by $\bar{M}=\int_{0}^{t} \bar{a}(s) d s$, where $\bar{a}: \mathbb{R}^{+} \rightarrow \mathbb{R}^{+}$is given by $\bar{a}(t)=\sup \{s: a(s) \leq t\}$ (see [1]).

The $N$-function $M$ is said to satisfy the $\Delta_{2}$-condition, written $M \in \Delta_{2}$, if for some $k>0$,

$$
M(2 t) \leq k M(t), \quad \forall t \geq 0 ;
$$

when (2.1) holds only for $t \geq$ some $t_{0}>0$ then $M$ is said to satisfy the $\Delta_{2}$-condition near infinity.

We will extend these $N$-functions to even functions on all $\mathbb{R}$.

Let $P$ and $Q$ be two $N$-functions. $P \ll Q$ means that $P$ grows essentially less rapidly than $Q$, i.e. for each $\varepsilon>0, P(t) / Q(\varepsilon t) \rightarrow 0$ as $t \rightarrow \infty$. This is the case if and only if $\lim _{t \rightarrow \infty} Q^{-1}(t) / P^{-1}(t)=0$.

2.2. Let $\Omega$ be an open subset of $\mathbb{R}^{N}$. The Orlicz class $K_{M}(\Omega)$ (resp. the Orlicz space $L_{M}(\Omega)$ ) is defined as the set of (equivalence classes of) real-valued measurable functions $u$ on $\Omega$ such that

$$
\int_{\Omega} M(u(x)) d x<\infty \quad\left(\text { resp. } \int_{\Omega} M(u(x) / \lambda) d x<\infty \text { for some } \lambda>0\right) .
$$

$L_{M}(\Omega)$ is a Banach space under the norm

$$
\|u\|_{M, \Omega}=\inf \left\{\lambda>0: \int_{\Omega} M(u(x) / \lambda) d x \leq 1\right\}
$$

and $K_{M}(\Omega)$ is a convex subset of $L_{M}(\Omega)$.

The closure in $L_{M}(\Omega)$ of the set of bounded measurable functions with compact support in $\bar{\Omega}$ is denoted by $E_{M}(\Omega)$.

The equality $E_{M}(\Omega)=L_{M}(\Omega)$ holds if and only if $M$ satisfies the $\Delta_{2^{-}}$ condition, for all $t$ or for $t$ large, according to whether $\Omega$ has infinite measure or not.

The dual of $E_{M}(\Omega)$ can be identified with $L_{\bar{M}}(\Omega)$ by means of the pairing $\int_{\Omega} u v d x$, and the dual norm of $L_{\bar{M}}(\Omega)$ is equivalent to $\|\cdot\|_{\bar{M}, \Omega}$. The space $L_{M}(\Omega)$ is reflexive if and only if $M$ and $\bar{M}$ satisfy the $\Delta_{2}$-condition, for all $t$ or for $t$ large, according to whether $\Omega$ has infinite measure or not. 
2.3. We now turn to the Orlicz-Sobolev space. $W^{1} L_{M}(\Omega)$ [resp. $\left.W^{1} E_{M}(\Omega)\right]$ is the space of all functions $u$ such that $u$ and its distributional derivatives up to order 1 lie in $L_{M}(\Omega)$ [resp. $\left.E_{M}(\Omega)\right]$. It is a Banach space under the norm

$$
\|u\|_{1, M}=\sum_{|\alpha| \leq 1}\left\|D^{\alpha} u\right\|_{M} .
$$

Thus, $W^{1} L_{M}(\Omega)$ and $W^{1} E_{M}(\Omega)$ can be identified with subspaces of the product of $N+1$ copies of $L_{M}(\Omega)$. Denoting this product by $\prod L_{M}$, we will use the weak topologies $\sigma\left(\prod L_{M}, \prod E_{\bar{M}}\right)$ and $\sigma\left(\prod L_{M}, \prod L_{\bar{M}}\right)$.

The space $W_{0}^{1} E_{M}(\Omega)$ is defined as the (norm) closure of the Schwartz space $\mathcal{D}(\Omega)$ in $W^{1} E_{M}(\Omega)$, and the space $W_{0}^{1} L_{M}(\Omega)$ as the $\sigma\left(\prod L_{M}, \prod E_{\bar{M}}\right)$ closure of $\mathcal{D}(\Omega)$ in $W^{1} L_{M}(\Omega)$.

We say that $u_{n}$ converges to $u$ for the modular convergence in $W^{1} L_{M}(\Omega)$ if for some $\lambda>0$,

$$
\int_{\Omega} M\left(\frac{D^{\alpha} u_{n}-D^{\alpha} u}{\lambda}\right) d x \rightarrow 0 \quad \text { for all }|\alpha| \leq 1 .
$$

This implies convergence for $\sigma\left(\prod L_{M}, \prod L_{\bar{M}}\right)$. If $M$ satisfies the $\Delta_{2}$-condition on $\mathbb{R}^{+}$, then modular convergence coincides with norm convergence.

2.4. Let $W^{-1} L_{\bar{M}}(\Omega)$ [resp. $W^{-1} E_{\bar{M}}(\Omega)$ ] denote the space of distributions on $\Omega$ which can be written as sums of derivatives of order $\leq 1$ of functions in $L_{\bar{M}}$ [resp. $\left.E_{\bar{M}}(\Omega)\right]$. It is a Banach space under the usual quotient norm.

If the open set $\Omega$ has the segment property then the space $\mathcal{D}(\Omega)$ is dense in $W_{0}^{1} L_{M}(\Omega)$ for the modular convergence and thus for the topology $\sigma\left(\prod L_{M}, \prod L_{\bar{M}}\right)$ (cf. $\left.[15,16]\right)$. Consequently, the action of a distribution in $W^{-1} L_{\bar{M}}(\Omega)$ on an element of $W_{0}^{1} L_{M}(\Omega)$ is well defined.

2.5. We recall some lemmas introduced in [5] which will be used in this paper.

Lemma 2.1. Let $F: \mathbb{R} \rightarrow \mathbb{R}$ be uniformly Lipschitzian, with $F(0)=0$. Let $M$ be an $N$-function and let $u \in W^{1} L_{M}(\Omega)$ (resp. $W^{1} E_{M}(\Omega)$ ). Then $F(u) \in W^{1} L_{M}(\Omega)\left(\right.$ resp. $\left.W^{1} E_{M}(\Omega)\right)$. Moreover, if the set $D$ of discontinuity points of $F^{\prime}$ is finite, then

$$
\frac{\partial}{\partial x_{i}} F(u)= \begin{cases}F^{\prime}(u) \frac{\partial}{\partial x_{i}} u & \text { a.e. in }\{x \in \Omega: u(x) \notin D\}, \\ 0 & \text { a.e. in }\{x \in \Omega: u(x) \in D\} .\end{cases}
$$

Lemma 2.2. Let $F: \mathbb{R} \rightarrow \mathbb{R}$ be uniformly Lipschitzian with $F(0)=0$. Suppose that the set of discontinuity points of $F^{\prime}$ is finite. Let $M$ be an $N$-function. Then the mapping $F: W^{1} L_{M}(\Omega) \rightarrow W^{1} L_{M}(\Omega)$ is sequentially continuous with respect to the weak* topology $\sigma\left(\prod L_{M}, \prod E_{\bar{M}}\right)$. 
2.6. We now give the following lemma which concerns operators of the Nemytskiu type in Orlicz spaces (see [5]).

Lemma 2.3. Let $\Omega$ be an open subset of $\mathbb{R}^{N}$ with finite measure. Let $M, P$ and $Q$ be $N$-functions such that $Q \ll P$, and let $f: \Omega \times \mathbb{R} \rightarrow \mathbb{R}$ be a Carathéodory function such that for a.e. $x \in \Omega$ and all $s \in \mathbb{R}$,

$$
|f(x, s)| \leq c(x)+k_{1} P^{-1} M\left(k_{2}|s|\right),
$$

where $k_{1}, k_{2}$ are real constants and $c(x) \in E_{Q}(\Omega)$. Then the Nemytskiu operator $N_{f}$ defined by $N_{f}(u)(x)=f(x, u(x))$ is strongly continuous from $\mathcal{P}\left(E_{M}(\Omega), 1 / k_{2}\right)=\left\{u \in L_{M}(\Omega): d\left(u, E_{M}(\Omega)\right)<1 / k_{2}\right\}$ into $E_{Q}(\Omega)$.

3. The main result. Let $\Omega$ be an open bounded subset of $\mathbb{R}^{N}, N \geq 2$, with the segment property.

Let $M$ be an $N$-function satisfying the $\Delta_{2}$-condition near infinity and let $P$ be an $N$-function such that $P \ll M$. Let $A(u)=-\operatorname{div}(a(x, u, \nabla u))$ be a Leray-Lions operator defined on $D(A) \subset W_{0}^{1} L_{M}(\Omega)$ into $W^{-1} L_{\bar{M}}(\Omega)$ where $a: \Omega \times \mathbb{R} \times \mathbb{R}^{N} \rightarrow \mathbb{R}^{N}$ is a Carathéodory function such that for a.e. $x \in \Omega$ and for all $\zeta, \zeta^{\prime} \in \mathbb{R}^{N}\left(\zeta \neq \zeta^{\prime}\right)$ and all $s \in \mathbb{R}$,

$$
\begin{gathered}
|a(x, s, \zeta)| \leq h(x)+k_{1} \bar{P}^{-1} M\left(k_{2}|s|\right)+k_{3} \bar{M}^{-1} M\left(k_{4}|\zeta|\right) \\
\left(a(x, s, \zeta)-a\left(x, s, \zeta^{\prime}\right)\right)\left(\zeta-\zeta^{\prime}\right)>0 \\
a(x, s, \zeta) \zeta \geq \alpha M(|\zeta| / \lambda)
\end{gathered}
$$

with $\alpha, \lambda>0, k_{1}, k_{2}, k_{3}, k_{4} \geq 0, h \in E_{\bar{M}}(\Omega)$.

Furthermore let $g: \Omega \times \mathbb{R} \times \mathbb{R}^{N} \rightarrow \mathbb{R}$ be a Carathéodory function such that for a.e. $x \in \Omega$ and for all $s \in \mathbb{R}$ and all $\zeta \in \mathbb{R}^{N}$,

$$
\begin{gathered}
g(x, s, \zeta) s \geq 0 \\
|g(x, s, \zeta)| \leq b(|s|)(c(x)+M(|\zeta| / \mu)),
\end{gathered}
$$

where $b: \mathbb{R}_{+} \rightarrow \mathbb{R}$ is a continuous nondecreasing function, $c$ is a given nonnegative function in $L^{1}(\Omega)$, and $\mu>0$. Finally, we assume that

$$
f \in L^{1}(\Omega) .
$$

Consider the following Dirichlet problem:

$$
A(u)+g(x, u, \nabla u)=f \quad \text { in } \Omega .
$$

We define $T_{0}^{1, M}(\Omega)$ to be the set of measurable functions $u: \Omega \rightarrow \mathbb{R}$ such that $T_{k}(u) \in W_{0}^{1} L_{M}(\Omega)$, where

$$
T_{k}(s)=\max (-k, \min (k, s)) \quad \text { for } s \in \mathbb{R} \text { and } k \geq 0 .
$$

We shall prove the following existence theorem. 
TheOREM 3.1. Assume that (3.1)-(3.6) hold true. Then there exists at least one solution of (3.7) in the following sense:

$$
\left\{\begin{array}{l}
u \in T_{0}^{1, M}(\Omega), \quad g(x, u, \nabla u) \in L^{1}(\Omega), \\
\int_{\Omega} a(x, u, \nabla u) \nabla T_{k}(u-v) d x+\int_{\Omega} g(x, u, \nabla u) T_{k}(u-v) d x \\
\leq \int_{\Omega} f T_{k}(u-v) d x, \quad \forall v \in W_{0}^{1} L_{M}(\Omega) \cap L^{\infty}(\Omega), \forall k>0 .
\end{array}\right.
$$

REMARK 3.1. Our result covers the critical case $M(t)=|t| \log (1+|t|)$ which satisfies the $\Delta_{2}$-condition but $\bar{M} \notin \Delta_{2}$.

If $M(t)=|t|^{p} / p$, we obtain the result given in [21].

REMARK 3.2. If $u$ is a solution of $(\mathrm{P})$ such that $a(x, u, \nabla u) \in L^{1}(\Omega)$, then it is also a solution of (3.7) in the distributional sense. This is the case, for example, if we take $a(x, s, \zeta)=a(x, s)|\zeta|^{p-2} \zeta \log ^{\beta}(1+|\zeta|)$ with $a(x, s)$ a Carathéodory function satisfying

$$
\alpha \leq a(x, s) \leq \gamma \quad \text { for a.e. } x \in \Omega \text { and all } s \in \mathbb{R}
$$

where $\alpha, \beta, \gamma>0$. Indeed, by choosing $0<\varepsilon<\frac{1}{\beta} \frac{p-1}{N-1}$, it is easy to see that there exists $C_{\varepsilon}>0$ such that

$$
\log ^{\beta}(1+|\zeta|) \leq C_{\varepsilon}|\zeta|^{\beta \varepsilon} \quad \text { for }|\zeta| \text { large enough, }
$$

and so that

$$
\int_{\Omega}|a(x, u, \nabla u)| d x \leq \gamma C_{\varepsilon} \int_{\Omega}|\nabla u|^{p-1+\beta \varepsilon} d x+C .
$$

In view of the fact that $p-1+\beta \varepsilon<N(p-1) /(N-1)$ one easily sees that $a(x, u, \nabla u) \in L^{1}(\Omega)$.

\section{Proof of Theorem 3.1}

STEP 1: A priori estimates. Consider the approximate problems:

$$
\left\{\begin{array}{c}
u_{n} \in W_{0}^{1} L_{M}(\Omega), g\left(x, u_{n}, \nabla u_{n}\right) \in L^{1}(\Omega), g\left(x, u_{n}, \nabla u_{n}\right) u_{n} \in L^{1}(\Omega), \\
\left\langle A\left(u_{n}\right), v\right\rangle+\int_{\Omega} g\left(x, u_{n}, \nabla u_{n}\right) v d x=\int_{\Omega} f_{n} v d x, \\
\forall v \in W_{0}^{1} L_{M}(\Omega) \cap L^{\infty}(\Omega),
\end{array}\right.
$$

where $f_{n}$ is a sequence of smooth functions which converges strongly to $f$ in $L^{1}(\Omega)$. By Theorem 3.1 of [5], there exists at least one solution $u_{n}$ of $(3.8)$. Taking $v=T_{k}\left(u_{n}\right)$ as test function in (3.8) gives

$$
\int_{\Omega} a\left(x, u_{n}, \nabla u_{n}\right) \nabla T_{k}\left(u_{n}\right) d x+\int_{\Omega} g\left(x, u_{n}, \nabla u_{n}\right) T_{k}\left(u_{n}\right) d x=\int_{\Omega} f_{n} T_{k}\left(u_{n}\right) d x
$$


and by using the fact that $g\left(x, u_{n}, \nabla u_{n}\right) T_{k}\left(u_{n}\right) \geq 0$, we obtain

$$
\int_{\left\{\left|u_{n}\right| \leq k\right\}} a\left(x, u_{n}, \nabla u_{n}\right) \nabla u_{n} d x \leq C k .
$$

Thanks to (3.3), one easily has

$$
\alpha \int_{\Omega} M\left(\left|\nabla T_{k}\left(u_{n}\right)\right| / \lambda\right) d x \leq C k .
$$

On the other hand, thanks to Lemma 5.7 of [15], there exist two positive constants $c_{1}$ and $c_{2}$ such that

$$
\int_{\Omega} M\left(T_{k}\left(u_{n}\right)\right) d x \leq c_{1} \int_{\Omega} M\left(c_{2}\left|\nabla T_{k}\left(u_{n}\right)\right|\right) d x .
$$

By the $\Delta_{2}$-condition there exist another two positive constants $c_{1}^{\prime}$ and $c_{2}^{\prime}$ such that

$$
M\left(c_{2} t\right) \leq c_{1}^{\prime}+c_{2}^{\prime} M(t / \lambda) \quad \text { for all } t \geq 0 .
$$

We then deduce, by using (3.9) and (3.10), that

$$
\int_{\Omega} M\left(T_{k}\left(u_{n}\right)\right) d x \leq c_{3}^{\prime}+c_{4}^{\prime} k
$$

which implies

$$
M(k) \operatorname{meas}\left\{\left|u_{n}\right|>k\right\} \leq c_{3}^{\prime}+c_{4}^{\prime} k
$$

and finally

$$
\operatorname{meas}\left\{\left|u_{n}\right|>k\right\} \leq \frac{c_{3}^{\prime}+c_{4}^{\prime} k}{M(k)}, \quad \forall n \text { and } \forall k>0 .
$$

We have, for every $\delta>0$,

$$
\begin{aligned}
\operatorname{meas}\left\{\left|u_{n}-u_{m}\right|>\delta\right\} \leq & \operatorname{meas}\left\{\left|u_{n}\right|>k\right\}+\operatorname{meas}\left\{\left|u_{m}\right|>k\right\} \\
& +\operatorname{meas}\left\{\left|T_{k}\left(u_{n}\right)-T_{k}\left(u_{m}\right)\right|>\delta\right\}
\end{aligned}
$$

Since $T_{k}\left(u_{n}\right)$ is bounded in $W_{0}^{1} L_{M}(\Omega)$, there exists some $v_{k} \in W_{0}^{1} L_{M}(\Omega)$ such that

$$
\begin{aligned}
T_{k}\left(u_{n}\right) \rightarrow v_{k} \quad & \text { weakly in } W_{0}^{1} L_{M}(\Omega) \text { for } \sigma\left(\prod L_{M}, \prod E_{\bar{M}}\right), \\
& \text { strongly in } E_{M}(\Omega),
\end{aligned}
$$

and almost everywhere in $\Omega$. Consequently, we can assume that $T_{k}\left(u_{n}\right)$ is a Cauchy sequence in measure in $\Omega$.

Let $\varepsilon>0$. Then, by (3.11) and (3.12), there exists some $k(\varepsilon)>0$ such that

$$
\operatorname{meas}\left\{\left|u_{n}-u_{m}\right|>\delta\right\} \leq \varepsilon
$$

for all $n, m \geq n_{0}(k(\varepsilon), \delta)$. This proves that $\left(u_{n}\right)$ is a Cauchy sequence in measure, thus $u_{n}$ converges almost everywhere to some measurable function $u$. 
Finally, by Lemma 4.4 of [15], we obtain

$$
\begin{aligned}
T_{k}\left(u_{n}\right) \rightarrow T_{k}(u) \quad & \text { weakly in } W_{0}^{1} L_{M}(\Omega) \text { for } \sigma\left(\prod L_{M}, \prod E_{\bar{M}}\right), \\
& \text { strongly in } E_{M}(\Omega) .
\end{aligned}
$$

Let $Q$ be an $N$-function such that $M \ll Q$ and the continuous embedding $W_{0}^{1} L_{M}(\Omega) \subset E_{Q}(\Omega)$ holds (see [15]). Let $\varepsilon>0$. Then there exists $C_{\varepsilon}>0$, as in [6], such that

$$
|a(x, s, \zeta)| \leq h(x)+C_{\varepsilon}+k_{1} \bar{M}^{-1} Q(\varepsilon|s|)+k_{3} \bar{M}^{-1} M(\varepsilon|\zeta|)
$$

for a.e. $x \in \Omega$ and for all $(s, \zeta) \in \mathbb{R} \times \mathbb{R}^{N}$. From (3.9) and (3.13) we deduce that $\left(a\left(x, T_{k}\left(u_{n}\right), \nabla T_{k}\left(u_{n}\right)\right)_{n}\right.$ is bounded in $\left(L_{\bar{M}}(\Omega)\right)^{N}$.

STEP 2: Almost everywhere convergence of the gradients. Fix $r, k>0$ and define $\Omega_{r}=\left\{x \in \Omega:\left|\nabla T_{k}(u(x))\right| \leq r\right\}$. We denote by $\chi_{r}$ the characteristic function of $\Omega_{r}$. Consider now, as in [9],

$$
\begin{aligned}
& I_{n, r}= \\
& \int_{\Omega_{r}}\left\{\left[a\left(x, T_{k}\left(u_{n}\right), \nabla T_{k}\left(u_{n}\right)\right)-a\left(x, T_{k}\left(u_{n}\right), \nabla T_{k}(u)\right)\right]\left[\nabla T_{k}\left(u_{n}\right)-\nabla T_{k}(u)\right]\right\}^{\theta} d x
\end{aligned}
$$

where $0<\theta<1$. Let $A_{n}$ be the expression in braces above. Then for any $\eta>0$,

$$
I_{n, r}=\int_{\Omega_{r} \cap\left\{\left|T_{k}\left(u_{n}\right)-T_{k}(u)\right| \leq \eta\right\}} A_{n}^{\theta} d x+\int_{\Omega_{r} \cap\left\{\left|T_{k}\left(u_{n}\right)-T_{k}(u)\right|>\eta\right\}} A_{n}^{\theta} d x .
$$

From (3.13) we deduce that $A_{n}$ is bounded in $L^{1}(\Omega)$ and by applying the Hölder inequality we obtain

$$
\begin{aligned}
I_{n, r} \leq & C_{1}\left\{\int_{\Omega_{r} \cap\left\{\left|T_{k}\left(u_{n}\right)-T_{k}(u)\right| \leq \eta\right\}} A_{n} d x\right\}^{\theta} \\
& +C_{2} \operatorname{meas}\left\{x:\left|T_{k}\left(u_{n}\right)-T_{k}(u)\right|>\eta\right\}^{1-\theta} .
\end{aligned}
$$

On the other hand, for any $s \geq r$ we have

$$
\begin{aligned}
& \quad \int_{\Omega_{r} \cap\left\{\left|T_{k}\left(u_{n}\right)-T_{k}(u)\right| \leq \eta\right\}} A_{n} d x \\
& \leq \int_{\left\{\left|T_{k}\left(u_{n}\right)-T_{k}(u)\right| \leq \eta\right\}}\left[a\left(x, T_{k}\left(u_{n}\right), \nabla T_{k}\left(u_{n}\right)\right)-a\left(x, T_{k}\left(u_{n}\right), \nabla T_{k}(u) \chi_{s}\right)\right] \\
& \left.\times\left[\nabla T_{k}\left(u_{n}\right)-\nabla T_{k}(u) \chi_{s}\right)\right] d x \\
& \leq \int_{\left\{\left|T_{k}\left(u_{n}\right)-T_{k}(u)\right| \leq \eta\right\}} a\left(x, T_{k}\left(u_{n}\right), \nabla T_{k}\left(u_{n}\right)\right)\left(\nabla T_{k}\left(u_{n}\right)-\nabla T_{k}(u) \chi_{s}\right) d x \\
& -\int_{\left\{\left|T_{k}\left(u_{n}\right)-T_{k}(u)\right| \leq \eta\right\}} a\left(x, T_{k}\left(u_{n}\right), \nabla T_{k}(u) \chi_{s}\right)\left(\nabla T_{k}\left(u_{n}\right)-\nabla T_{k}(u) \chi_{s}\right) d x
\end{aligned}
$$




$$
\begin{aligned}
= & \int_{\Omega} a\left(x, T_{k}\left(u_{n}\right), \nabla T_{k}\left(u_{n}\right)\right) \nabla T_{\eta}\left(T_{k}\left(u_{n}\right)-T_{k}(u)\right) d x \\
& +\int_{\Omega} a\left(x, T_{k}\left(u_{n}\right), \nabla T_{k}\left(u_{n}\right)\right) \nabla T_{k}(u) \chi_{\Omega \backslash \Omega_{s}} \chi_{\left\{\left|T_{k}\left(u_{n}\right)-T_{k}(u)\right| \leq \eta\right\}} d x \\
& -\int_{\left\{\left|T_{k}\left(u_{n}\right)-T_{k}(u)\right| \leq \eta\right\}} a\left(x, T_{k}\left(u_{n}\right), \nabla T_{k}(u) \chi_{s}\right)\left(\nabla T_{k}\left(u_{n}\right)-\nabla T_{k}(u) \chi_{s}\right) d x .
\end{aligned}
$$

The use of the test function $T_{\eta}\left(u_{n}-T_{k}(u)\right)$ in (3.8) gives

$$
\begin{array}{r}
\left\langle A\left(u_{n}\right), T_{\eta}\left(u_{n}-T_{k}(u)\right)\right\rangle+\int_{\Omega} g\left(x, u_{n}, \nabla u_{n}\right) T_{\eta}\left(u_{n}-T_{k}(u) d x\right. \\
\leq \int_{\Omega} f_{n} T_{\eta}\left(u_{n}-T_{k}(u) d x,\right.
\end{array}
$$

which implies

$$
\left\langle A\left(u_{n}\right), T_{\eta}\left(u_{n}-T_{k}(u)\right)\right\rangle \leq C \eta
$$

Note that

$$
\begin{aligned}
& \left\langle A\left(u_{n}\right), T_{\eta}\left(u_{n}-T_{k}(u)\right)\right\rangle \\
& \geq \int_{\Omega} a\left(x, T_{k}\left(u_{n}\right), \nabla T_{k}\left(u_{n}\right)\right) \nabla T_{\eta}\left(T_{k}\left(u_{n}\right)-T_{k}(u)\right) d x \\
& \quad-\int_{\left|u_{n}\right|>k}\left|a\left(x, T_{k+\eta}\left(u_{n}\right), \nabla T_{k+\eta}\left(u_{n}\right)\right)\right|\left|\nabla T_{k}(u)\right| d x .
\end{aligned}
$$

We denote by $\varepsilon_{i}^{\eta}(n)(i=1,2, \ldots)$ various sequences of real numbers which tend to 0 as $n \rightarrow \infty$ for $\eta$ fixed.

The second term of the right hand side of (3.18) tends to zero since $a\left(x, T_{k+\eta}\left(u_{n}\right), \nabla T_{k+\eta}\left(u_{n}\right)\right)$ is bounded in $\left(L_{\bar{M}}(\Omega)\right)^{N}$ while $\chi_{\left\{\left|u_{n}\right|>k\right\}}\left|\nabla T_{k}(u)\right|$ $\rightarrow 0$ strongly in $\left(E_{M}(\Omega)\right)^{N}$. Consequently, from (3.17) and (3.18), we have

$$
\int_{\Omega} a\left(x, T_{k}\left(u_{n}\right), \nabla T_{k}\left(u_{n}\right)\right) \nabla T_{\eta}\left(T_{k}\left(u_{n}\right)-T_{k}(u)\right) d x \leq C \eta+\varepsilon_{1}^{\eta}(n) .
$$

Since $a\left(x, T_{k}\left(u_{n}\right), \nabla T_{k}\left(u_{n}\right)\right) \chi_{\left\{\left|T_{k}\left(u_{n}\right)-T_{k}(u)\right| \leq \eta\right\}}$ is bounded in $\left(L_{\bar{M}}(\Omega)\right)^{N}$ it follows that $a\left(x, T_{k}\left(u_{n}\right), \nabla T_{k}\left(u_{n}\right)\right) \chi_{\left\{\left|T_{k}\left(u_{n}\right)-T_{k}(u)\right| \leq \eta\right\}}$ converges to $h$ weakly in $\left(L_{\bar{M}}(\Omega)\right)^{N}$ for $\sigma\left(\prod L_{\bar{M}}, \prod E_{M}(\Omega)\right)$, for some $h \in\left(L_{\bar{M}}(\Omega)\right)^{N}$. We deduce that the second term of the right hand side of (3.15) tends to

$$
\int_{\Omega \backslash \Omega_{s}} h \nabla T_{k}(u) d x \quad \text { as } n \rightarrow \infty .
$$

The third term of the right hand side of (3.15) tends to 0 since $a\left(x, T_{k}\left(u_{n}\right), \nabla T_{k}(u) \chi_{s}\right) \chi_{\left\{\left|T_{k}\left(u_{n}\right)-T_{k}(u)\right| \leq \eta\right\}}$ converges strongly to $a\left(x, T_{k}(u)\right.$, $\left.\nabla T_{k}(u) \chi_{s}\right)$ in $E_{\bar{M}}(\Omega)^{N}$ by Lemma 2.3 while $\nabla T_{k}\left(u_{n}\right)$ tends weakly to 
$\nabla T_{k}(u)$ and

$$
\int_{\Omega} a\left(x, T_{k}(u), \nabla T_{k}(u) \chi_{s}\right)\left[\nabla T_{k}(u)-\nabla T_{k}(u) \chi_{s}\right] d x=0 .
$$

Finally, from (3.15) and in view of (3.19) we have

$$
\begin{aligned}
I_{n, r} \leq & C_{1}\left(C \eta+\varepsilon_{2}^{\eta}(n)+\int_{\Omega} h \nabla T_{k}(u) \chi_{\Omega \backslash \Omega_{s}} d x\right)^{\theta} \\
& +C_{2} \operatorname{meas}\left\{x:\left|T_{k}\left(u_{n}\right)-T_{k}(u)\right|>\eta\right\}^{1-\theta},
\end{aligned}
$$

which gives, by passing to the limsup over $n$,

$$
\limsup _{n \rightarrow \infty} I_{n, r} \leq C\left(\eta+\int_{\Omega} h \nabla T_{k}(u) \chi_{\Omega \backslash \Omega_{s}} d x\right)^{\theta} .
$$

Then by, letting $s \rightarrow \infty$ and choosing $\eta$ small enough, we obtain

$$
\lim _{n \rightarrow \infty} I_{n, r}=0
$$

and so, as in [4],

$$
\nabla u_{n} \rightarrow \nabla u \quad \text { a.e. in } \Omega \text {. }
$$

STEP 3: Strong convergence of $M\left(\left|\nabla T_{k}\left(u_{n}\right)\right| / \mu\right)$ in $L^{1}(\Omega)$ (i.e. modular convergence of $\nabla T_{k}\left(u_{n}\right)$ in $\left.\left(L_{M}(\Omega)\right)^{N}\right)$. Now fix $k>0$, and let

$$
\gamma=\left(K \frac{b(k)}{2 \alpha}\right)^{2}, \quad \phi(s)=s \exp \left(\gamma s^{2}\right) \text {. }
$$

It is well known that

$$
\phi^{\prime}(s)-K \frac{b(k)}{\alpha}|\phi(s)| \geq \frac{1}{2}, \quad \forall s \in \mathbb{R},
$$

where $K$ is a constant which will be used later.

Consider now the function $h_{m}, m>0$, defined by

$$
h_{m}(t)= \begin{cases}1 & \text { if }|t| \leq m \\ -(t / m) \operatorname{sgn}(t)+2 & \text { if } m \leq|t| \leq 2 m \\ 0 & \text { if }|t|>2 m\end{cases}
$$

Let $v_{n, m}=h_{m}\left(u_{n}\right) \phi\left(z_{n}\right)$ with $z_{n}=T_{k}\left(u_{n}\right)-T_{k}(u)$. The use of $v_{n, m}$ as test function in (3.8) gives

$$
\begin{aligned}
\left\langle A\left(u_{n}\right), h_{m}\left(u_{n}\right) \phi\left(z_{n}\right)\right\rangle+\int_{\Omega} g\left(x, u_{n}, \nabla u_{n}\right) h_{m}\left(u_{n}\right) \phi\left(z_{n}\right) d x & \\
& =\int_{\Omega} f_{n} h_{m}\left(u_{n}\right) \phi\left(z_{n}\right) d x .
\end{aligned}
$$

It follows that 


$$
\begin{aligned}
\int_{\Omega} a(x, & \left.u_{n}, \nabla u_{n}\right)\left[\nabla T_{k}\left(u_{n}\right)-\nabla T_{k}(u)\right] h_{m}\left(u_{n}\right) \phi^{\prime}\left(z_{n}\right) d x \\
& +\int_{\Omega} a\left(x, u_{n}, \nabla u_{n}\right) \nabla u_{n} h_{m}^{\prime}\left(u_{n}\right) \phi\left(z_{n}\right) d x \\
& +\int_{\Omega} g\left(x, u_{n}, \nabla u_{n}\right) h_{m}\left(u_{n}\right) \phi\left(z_{n}\right) d x \leq \int_{\Omega} f_{n} h_{m}\left(u_{n}\right) \phi\left(z_{n}\right) d x .
\end{aligned}
$$

Denote by $\varepsilon_{m}^{1}(n), \varepsilon_{m}^{2}(n), \ldots$ various sequences of real numbers which converge to zero as $n$ tends to infinity for any fixed value of $m$.

Since $g\left(x, u_{n}, \nabla u_{n}\right) h_{m}\left(u_{n}\right) \phi\left(z_{n}\right) \geq 0$ on the subset $\left\{x \in \Omega:\left|u_{n}(x)\right|>k\right\}$, we deduce from (3.22) that

$$
\begin{aligned}
& \quad \int_{\Omega} a\left(x, u_{n}, \nabla u_{n}\right)\left[\nabla T_{k}\left(u_{n}\right)-\nabla T_{k}(u)\right] h_{m}\left(u_{n}\right) \phi^{\prime}\left(z_{n}\right) d x \\
& +\int_{\Omega} a\left(x, u_{n}, \nabla u_{n}\right) \nabla u_{n} h_{m}^{\prime}\left(u_{n}\right) \phi\left(z_{n}\right) d x \\
& +\int_{\left\{\left|u_{n}\right| \leq k\right\}} g\left(x, u_{n}, \nabla u_{n}\right) h_{m}\left(u_{n}\right) \phi\left(z_{n}\right) d x \leq \int_{\Omega} f_{n} h_{m}\left(u_{n}\right) \phi\left(z_{n}\right) d x=\varepsilon_{m}^{1}(n) .
\end{aligned}
$$

The first term of the left hand side of (3.23) can be written as

$$
\begin{aligned}
\int_{\Omega} a\left(x, u_{n}, \nabla u_{n}\right)\left[\nabla T_{k}\left(u_{n}\right)-\nabla T_{k}(u)\right] h_{m}\left(u_{n}\right) \phi^{\prime}\left(z_{n}\right) d x \\
=\int_{\left\{\left|u_{n}\right| \leq k\right\}} a\left(x, u_{n}, \nabla u_{n}\right)\left[\nabla T_{k}\left(u_{n}\right)-\nabla T_{k}(u)\right] h_{m}\left(u_{n}\right) \phi^{\prime}\left(z_{n}\right) d x \\
\quad-\int_{\left\{\left|u_{n}\right|>k\right\}} a\left(x, u_{n}, \nabla u_{n}\right) \nabla T_{k}(u) h_{m}\left(u_{n}\right) \phi^{\prime}\left(z_{n}\right) d x .
\end{aligned}
$$

For the second term of the right hand side of (3.24), we have

$$
\begin{aligned}
& \left|\int_{\left\{\left|u_{n}\right|>k\right\}} a\left(x, u_{n}, \nabla u_{n}\right) \nabla T_{k}(u) h_{m}\left(u_{n}\right) \phi^{\prime}\left(z_{n}\right) d x\right| \\
& \leq C_{k} \int_{\Omega}\left|a\left(x, T_{2 m}\left(u_{n}\right), \nabla T_{2 m}\left(u_{n}\right)\right)\right|\left|\nabla T_{k}(u)\right| \chi_{\left\{\left|u_{n}\right|>k\right\}} d x
\end{aligned}
$$

where $C_{k}=\phi^{\prime}(2 k)$. The right hand side of the last inequality tends to 0 as $n$ tends to infinity. Indeed, the sequence $\left(a\left(x, T_{2 m}\left(u_{n}\right), \nabla T_{2 m}\left(u_{n}\right)\right)\right)_{n}$ is bounded in $\left(L_{\bar{M}}(\Omega)\right)^{N}$ while $\nabla T_{k}(u) \chi_{\left\{\left|u_{n}\right|>k\right\}}$ tends to 0 strongly in $\left(E_{M}(\Omega)\right)^{N}$.

For the first term of the right hand side of (3.24), we can write

$$
\begin{aligned}
\int_{\left\{\left|u_{n}\right| \leq k\right\}} a\left(x, u_{n},\right. & \left.\nabla u_{n}\right)\left[\nabla T_{k}\left(u_{n}\right)-\nabla T_{k}(u)\right] h_{m}\left(u_{n}\right) \phi^{\prime}\left(z_{n}\right) d x \\
= & \int_{\Omega}\left[a\left(x, T_{k}\left(u_{n}\right), \nabla T_{k}\left(u_{n}\right)\right)-a\left(x, T_{k}\left(u_{n}\right), \nabla T_{k}(u) \chi_{s}\right)\right] \\
& \times\left[\nabla T_{k}\left(u_{n}\right)-\nabla T_{k}(u) \chi_{s}\right] h_{m}\left(u_{n}\right) \phi^{\prime}\left(z_{n}\right) d x
\end{aligned}
$$




$$
\begin{aligned}
& +\int_{\Omega} a\left(x, T_{k}\left(u_{n}\right), \nabla T_{k}(u) \chi_{s}\right)\left[\nabla T_{k}\left(u_{n}\right)-\nabla T_{k}(u) \chi_{s}\right] h_{m}\left(u_{n}\right) \phi^{\prime}\left(z_{n}\right) d x \\
& -\int_{\Omega} a\left(x, T_{k}\left(u_{n}\right), \nabla T_{k}\left(u_{n}\right)\right) \nabla T_{k}(u) \chi_{\Omega \backslash \Omega_{s}} h_{m}\left(u_{n}\right) \phi^{\prime}\left(z_{n}\right) d x .
\end{aligned}
$$

The second term of the right hand side of (3.25) tends to 0 since

$$
\begin{array}{r}
a\left(x, T_{k}\left(u_{n}\right), \nabla T_{k}(u) \chi_{s}\right) h_{m}\left(u_{n}\right) \phi^{\prime}\left(z_{n}\right) \rightarrow a\left(x, T_{k}(u), \nabla T_{k}(u) \chi_{s}\right) h_{m}(u) \\
\text { strongly in }\left(E_{\bar{M}}(\Omega)\right)^{N}
\end{array}
$$

by Lemma 2.3 and

$\nabla T_{k}\left(u_{n}\right) \rightarrow \nabla T_{k}(u) \quad$ weakly in $\left(L_{M}(\Omega)\right)^{N}$ for $\sigma\left(\prod L_{M}(\Omega), \prod E_{\bar{M}}(\Omega)\right)$.

The third term of the right hand side of (3.25) tends to the quantity $-\int_{\Omega} a\left(x, T_{k}(u), \nabla T_{k}(u)\right) \nabla T_{k}(u) \chi_{\Omega \backslash \Omega_{s}} h_{m}(u) d x$ as $n \rightarrow \infty$ since

$$
\begin{aligned}
& a\left(x, T_{k}\left(u_{n}\right), \nabla T_{k}(u) \chi_{s}\right) \rightarrow a\left(x, T_{k}(u), \nabla T_{k}(u) \chi_{s}\right) \\
& \text { weakly for } \sigma\left(\prod E_{\bar{M}}(\Omega), \prod L_{M}(\Omega)\right) .
\end{aligned}
$$

Consequently, from (3.24) we have

$$
\begin{aligned}
\int_{\Omega} a(x, & \left.u_{n}, \nabla u_{n}\right)\left[\nabla T_{k}\left(u_{n}\right)-\nabla T_{k}(u)\right] h_{m}\left(u_{n}\right) \phi^{\prime}\left(z_{n}\right) d x \\
= & \int_{\Omega}\left[a\left(x, T_{k}\left(u_{n}\right), \nabla T_{k}\left(u_{n}\right)\right)-a\left(x, T_{k}\left(u_{n}\right), \nabla T_{k}(u) \chi_{s}\right)\right] \\
& \times\left[\nabla T_{k}\left(u_{n}\right)-\nabla T_{k}(u) \chi_{s}\right] h_{m}\left(u_{n}\right) \phi^{\prime}\left(z_{n}\right) d x \\
& -\int_{\Omega} a\left(x, T_{k}(u), \nabla T_{k}(u)\right) \nabla T_{k}(u) \chi_{\Omega \backslash \Omega_{s}} h_{m}(u) d x+\varepsilon_{m}^{2}(n) .
\end{aligned}
$$

On the other hand

$$
\begin{aligned}
& \left|\int_{\Omega} a\left(x, u_{n}, \nabla u_{n}\right) \nabla u_{n} h_{m}^{\prime}\left(u_{n}\right) \phi\left(z_{n}\right) d x\right| \\
& \leq \frac{2 \phi(2 k)}{m} \int_{\left\{m \leq\left|u_{n}\right| \leq 2 m\right\}} a\left(x, u_{n}, \nabla u_{n}\right) \nabla u_{n} d x
\end{aligned}
$$

and by using $T_{m}\left(u_{n}-T_{m}\left(u_{n}\right)\right)$ as test function in (3.8), we obtain

$$
\left|\int_{\Omega} a\left(x, u_{n}, \nabla u_{n}\right) \nabla u_{n} h_{m}^{\prime}\left(u_{n}\right) \phi\left(z_{n}\right) d x\right| \leq 2 \phi(2 k) \int_{\left\{\left|u_{n}\right| \geq m\right\}}\left|f_{n}\right| d x .
$$

Thanks to the $\Delta_{2}$-condition there exist two positive constants $K$ and $K^{\prime}$ such that

$$
M(t / \mu) \leq K M(t / \lambda)+K^{\prime}, \quad \forall t \geq 0 .
$$

If we denote by $J_{n, m}$ the third term of the left hand side of (3.23), then (3.28) yields 


$$
\begin{aligned}
&\left|J_{n, m}\right| \leq \int_{\left\{\left|u_{n}\right| \leq k\right\}} b(k)\left(c(x)+K^{\prime}+K M\left(\left|\nabla u_{n}\right| / \lambda\right)\right) h_{m}\left(u_{n}\right)\left|\phi\left(z_{n}\right)\right| d x \\
& \leq b(k) \int_{\Omega}\left(c(x)+K^{\prime}\right)\left|\phi\left(z_{n}\right)\right| d x \\
&+K \frac{b(k)}{\alpha} \int_{\Omega} a\left(x, T_{k}\left(u_{n}\right), \nabla T_{k}\left(u_{n}\right)\right) \nabla T_{k}\left(u_{n}\right) h_{m}\left(u_{n}\right)\left|\phi\left(z_{n}\right)\right| d x \\
& \leq \varepsilon_{m}^{3}(n)+K \frac{b(k)}{\alpha} \int_{\Omega}\left[a\left(x, T_{k}\left(u_{n}\right), \nabla T_{k}\left(u_{n}\right)\right)-a\left(x, T_{k}\left(u_{n}\right), \nabla T_{k}(u) \chi_{s}\right)\right] \\
& \quad \times\left[\nabla T_{k}\left(u_{n}\right)-\nabla T_{k}(u) \chi_{s}\right] h_{m}\left(u_{n}\right)\left|\phi\left(z_{n}\right)\right| d x .
\end{aligned}
$$

Indeed, we have

$$
\begin{aligned}
& \int_{\Omega} a\left(x, T_{k}\left(u_{n}\right), \nabla T_{k}\left(u_{n}\right)\right) \nabla T_{k}\left(u_{n}\right) h_{m}\left(u_{n}\right)\left|\phi\left(z_{n}\right)\right| d x \\
= & \int_{\Omega}\left[a\left(x, T_{k}\left(u_{n}\right), \nabla T_{k}\left(u_{n}\right)\right)-a\left(x, T_{k}\left(u_{n}\right), \nabla T_{k}(u) \chi_{s}\right)\right] \\
& \times\left[\nabla T_{k}\left(u_{n}\right)-\nabla T_{k}(u) \chi_{s}\right] h_{m}\left(u_{n}\right)\left|\phi\left(z_{n}\right)\right| d x \\
+ & \int_{\Omega} a\left(x, T_{k}\left(u_{n}\right), \nabla T_{k}\left(u_{n}\right)\right) \nabla T_{k}(u) \chi_{s} h_{m}\left(u_{n}\right)\left|\phi\left(z_{n}\right)\right| d x \\
+ & \int_{\Omega} a\left(x, T_{k}\left(u_{n}\right), \nabla T_{k}(u) \chi_{s}\right)\left[\nabla T_{k}\left(u_{n}\right)-\nabla T_{k}(u) \chi_{s}\right] h_{m}\left(u_{n}\right)\left|\phi\left(z_{n}\right)\right| d x
\end{aligned}
$$

It is easy to see that the second term of the right hand side of (3.30) tends to 0 as $n$ tends to infinity, since $\left(a\left(x, T_{k}\left(u_{n}\right), \nabla T_{k}\left(u_{n}\right)\right)\right)_{n}$ is bounded in $\left(L_{\bar{M}}(\Omega)\right)^{N}$, and

$$
\nabla T_{k}(u) \chi_{s} h_{m}\left(u_{n}\right)\left|\phi\left(z_{n}\right)\right| \rightarrow 0 \quad \text { strongly in }\left(E_{M}(\Omega)\right)^{N}
$$

by Lebesgue's theorem.

The third term of the right hand side of (3.30) also tends to 0 since

$$
a\left(x, T_{k}\left(u_{n}\right), \nabla T_{k}(u) \chi_{s}\right)\left|\phi\left(z_{n}\right)\right| \rightarrow 0 \quad \text { strongly in }\left(E_{\bar{M}}(\Omega)\right)^{N}
$$

by Lemma 2.3 while

$$
\left[\nabla T_{k}\left(u_{n}\right)-\nabla T_{k}(u) \chi_{s}\right] h_{m}\left(u_{n}\right) \quad \text { is bounded in }\left(L_{M}(\Omega)\right)^{N} .
$$

Combining (3.26) and (3.29) we obtain

$$
\begin{aligned}
& \int_{\Omega}\left[a\left(x, T_{k}\left(u_{n}\right), \nabla T_{k}\left(u_{n}\right)\right)-a\left(x, T_{k}\left(u_{n}\right), \nabla T_{k}(u) \chi_{s}\right)\right] \\
& \quad \times\left[\nabla T_{k}\left(u_{n}\right)-\nabla T_{k}(u) \chi_{s}\right] h_{m}\left(u_{n}\right)\left(\phi^{\prime}\left(z_{n}\right)-K \frac{b(k)}{\alpha}\left|\phi\left(z_{n}\right)\right|\right) d x \\
& \leq \varepsilon_{m}^{4}(n)-\int_{\Omega} a\left(x, T_{k}(u), \nabla T_{k}(u)\right) \nabla T_{k}(u) \chi_{\Omega \backslash \Omega_{s}} h_{m}(u) d x \\
& \quad+\phi(2 k) \int_{\left\{\left|u_{n}\right| \geq m\right\}}\left|f_{n}\right| d x
\end{aligned}
$$


which implies, by (3.21),

$$
\begin{aligned}
\int_{\Omega}\left[a \left(x, T_{k}\left(u_{n}\right), \nabla\right.\right. & \left.\left.T_{k}\left(u_{n}\right)\right)-a\left(x, T_{k}\left(u_{n}\right), \nabla T_{k}(u) \chi_{s}\right)\right] \\
& \times\left[\nabla T_{k}\left(u_{n}\right)-\nabla T_{k}(u) \chi_{s}\right] h_{m}\left(u_{n}\right) d x \\
\leq & 2 \varepsilon_{m}^{4}(n)-2 \int_{\Omega} a\left(x, T_{k}(u), \nabla T_{k}(u)\right) \nabla T_{k}(u) \chi_{\Omega \backslash \Omega_{s}} h_{m}(u) d x \\
& +4 \phi(2 k) \int_{\left\{\left|u_{n}\right| \geq m\right\}}\left|f_{n}\right| d x .
\end{aligned}
$$

Hence

$$
\begin{aligned}
& \int_{\Omega} a\left(x, T_{k}\left(u_{n}\right), \nabla T_{k}\left(u_{n}\right)\right) \nabla T_{k}\left(u_{n}\right) h_{m}\left(u_{n}\right) d x \\
& \leq \int_{\Omega} a\left(x, T_{k}\left(u_{n}\right), \nabla T_{k}\left(u_{n}\right)\right) \nabla T_{k}(u) \chi_{s} d x \\
& \quad+\int_{\Omega} a\left(x, T_{k}\left(u_{n}\right), \nabla T_{k}(u) \chi_{s}\right)\left[\nabla T_{k}\left(u_{n}\right)-\nabla T_{k}(u) \chi_{s}\right] h_{m}\left(u_{n}\right) d x \\
& \quad+2 \varepsilon_{m}^{4}(n)-2 \int_{\Omega} a\left(x, T_{k}(u), \nabla T_{k}(u)\right) \nabla T_{k}(u) \chi_{\Omega \backslash \Omega_{s}} h_{m}(u) d x \\
& \quad+4 \phi(2 k) \int_{\left\{\left|u_{n}\right| \geq m\right\}}\left|f_{n}\right| d x .
\end{aligned}
$$

By passing to the limsup over $n$, one has

$$
\begin{aligned}
& \quad \limsup _{n \rightarrow \infty} \int_{\Omega} a\left(x, T_{k}\left(u_{n}\right), \nabla T_{k}\left(u_{n}\right)\right) \nabla T_{k}\left(u_{n}\right) h_{m}\left(u_{n}\right) d x \\
& \leq \limsup _{n \rightarrow \infty} \int_{\Omega} a\left(x, T_{k}\left(u_{n}\right), \nabla T_{k}\left(u_{n}\right)\right) \nabla T_{k}(u) \chi_{s} h_{m}\left(u_{n}\right) d x \\
& +\limsup _{n \rightarrow \infty} \int_{\Omega} a\left(x, T_{k}\left(u_{n}\right), \nabla T_{k}(u) \chi_{s}\right)\left[\nabla T_{k}\left(u_{n}\right)-\nabla T_{k}(u) \chi_{s}\right] h_{m}\left(u_{n}\right) d x \\
& -2 \int_{\Omega} a\left(x, T_{k}(u), \nabla T_{k}(u)\right) \nabla T_{k}(u) \chi_{\Omega \backslash \Omega_{s}} h_{m}(u) d x \\
& +4 \phi(2 k) \int_{\{|u| \geq m\}}|f| d x .
\end{aligned}
$$

The second term of the right hand side of (3.33) tends to 0 , since $a\left(x, T_{k}\left(u_{n}\right), \nabla T_{k}(u) \chi_{s}\right) \rightarrow a\left(x, T_{k}(u), \nabla T_{k}(u) \chi_{s}\right)$ strongly in $E_{\bar{M}}(\Omega)$ while $\nabla T_{k}\left(u_{n}\right)$ tends weakly to $\nabla T_{k}(u)$.

The first term of the right hand side of (3.33) tends to

$$
\int_{\Omega} a\left(x, T_{k}(u), \nabla T_{k}(u)\right) \nabla T_{k}(u) \chi_{s} h_{m}(u) d x
$$

since $a\left(x, T_{k}\left(u_{n}\right), \nabla T_{k}\left(u_{n}\right)\right) h_{m}\left(u_{n}\right) \rightarrow a\left(x, T_{k}(u), \nabla T_{k}(u)\right) h_{m}(u)$ weakly in 
$\left(L_{\bar{M}}(\Omega)\right)^{N}$ for $\sigma\left(\prod L_{\bar{M}}, \prod E_{M}\right)$ while $\nabla T_{k}(u) \chi_{s} \in E_{M}(\Omega)$. We deduce that $\limsup _{n \rightarrow \infty} \int_{\Omega} a\left(x, T_{k}(u), \nabla T_{k}(u)\right) \nabla T_{k}(u) h_{m}(u) d x$

$$
\begin{aligned}
\leq & \int_{\Omega} a\left(x, T_{k}(u), \nabla T_{k}(u)\right) \nabla T_{k}(u) \chi_{s} h_{m}(u) d x \\
& -2 \int_{\Omega} a\left(x, T_{k}(u), \nabla T_{k}(u)\right) \nabla T_{k}(u) \chi_{\Omega \backslash \Omega_{s}} h_{m}(u) d x \\
& +4 \phi(2 k) \int_{\{|u| \geq m\}}|f| d x .
\end{aligned}
$$

Passing again to the limsup but now over $m$, and using the fact that $a\left(x, T_{k}(u), \nabla T_{k}(u)\right) \nabla T_{k}(u) \in L^{1}(\Omega), f \in L^{1}(\Omega)$ and $h_{m}(u) \rightarrow 1$ as $m \rightarrow \infty$, one easily obtains by Lebesgue's theorem

$$
\begin{aligned}
\limsup _{m \rightarrow \infty} \limsup _{n \rightarrow \infty} \int_{\Omega} a\left(x, T_{k}\left(u_{n}\right)\right. & \left., \nabla T_{k}\left(u_{n}\right)\right) \nabla T_{k}\left(u_{n}\right) h_{m}\left(u_{n}\right) d x \\
\leq & \int_{\Omega} a\left(x, T_{k}(u), \nabla T_{k}(u)\right) \nabla T_{k}(u) \chi_{s} d x \\
& \quad-2 \int_{\Omega} a\left(x, T_{k}(u), \nabla T_{k}(u)\right) \nabla T_{k}(u) \chi_{\Omega \backslash \Omega_{s}} d x .
\end{aligned}
$$

Using again the fact that $a\left(x, T_{k}(u), \nabla T_{k}(u)\right) \nabla T_{k}(u) \in L^{1}(\Omega)$ and letting $s \rightarrow \infty$ we get, since meas $\left(\Omega \backslash \Omega_{s}\right) \rightarrow 0$,

$$
\begin{aligned}
\limsup _{m \rightarrow \infty} \limsup _{n \rightarrow \infty} \int_{\Omega} a\left(x, T_{k}\left(u_{n}\right), \nabla T_{k}\left(u_{n}\right)\right) \nabla T_{k}\left(u_{n}\right) h_{m}\left(u_{n}\right) d x & \\
& \leq \int_{\Omega} a\left(x, T_{k}(u), \nabla T_{k}(u)\right) \nabla T_{k}(u) d x .
\end{aligned}
$$

On the other hand, by Fatou's lemma,

$$
\begin{aligned}
& \int_{\Omega} a\left(x, T_{k}(u), \nabla T_{k}(u)\right) \nabla T_{k}(u) d x \\
& \quad \leq \limsup _{m \rightarrow \infty} \limsup _{n \rightarrow \infty} \int_{\Omega} a\left(x, T_{k}\left(u_{n}\right), \nabla T_{k}\left(u_{n}\right)\right) \nabla T_{k}\left(u_{n}\right) h_{m}\left(u_{n}\right) d x,
\end{aligned}
$$

which implies finally

$$
\begin{aligned}
\limsup _{m \rightarrow \infty} \limsup _{n \rightarrow \infty} \int_{\Omega} a\left(x, T_{k}\left(u_{n}\right),\right. & \left.\nabla T_{k}\left(u_{n}\right)\right) \nabla T_{k}\left(u_{n}\right) h_{m}\left(u_{n}\right) d x \\
= & \int_{\Omega} a\left(x, T_{k}(u), \nabla T_{k}(u)\right) \nabla T_{k}(u) d x .
\end{aligned}
$$


Taking now $\left(1-h_{m}\left(u_{n}\right)\right) T_{k}\left(u_{n}\right)$ as test function in (3.8) we obtain

$$
\begin{aligned}
\left\langle A\left(u_{n}\right),\left(1-h_{m}\left(u_{n}\right)\right) T_{k}\left(u_{n}\right)\right\rangle+\int_{\Omega} g\left(x, u_{n}, \nabla u_{n}\right) & \left.\left(1-h_{m}\left(u_{n}\right)\right) T_{k}\left(u_{n}\right)\right) d x \\
= & \int_{\Omega} f_{n}\left(1-h_{m}\left(u_{n}\right)\right) T_{k}\left(u_{n}\right) d x
\end{aligned}
$$

and thanks to the sign condition (3.4) we obtain

$$
\left\langle A\left(u_{n}\right),\left(1-h_{m}\left(u_{n}\right)\right) T_{k}\left(u_{n}\right)\right\rangle \leq \int_{\Omega} f_{n}\left(1-h_{m}\left(u_{n}\right)\right) T_{k}\left(u_{n}\right) d x
$$

and so

$$
\begin{aligned}
& \int_{\Omega} a\left(x, T_{k}\left(u_{n}\right), \nabla T_{k}\left(u_{n}\right)\right) \nabla T_{k}\left(u_{n}\right)\left(1-h_{m}\left(u_{n}\right)\right) d x \\
\leq & \int_{\Omega} f_{n}\left(1-h_{m}\left(u_{n}\right)\right) T_{k}\left(u_{n}\right) d x+\int_{\Omega} a\left(x, u_{n}, \nabla u_{n}\right) \nabla u_{n} h_{m}^{\prime}\left(u_{n}\right) T_{k}\left(u_{n}\right) d x .
\end{aligned}
$$

Since

$$
\int_{\Omega} a\left(x, u_{n}, \nabla u_{n}\right) \nabla u_{n} h_{m}^{\prime}\left(u_{n}\right) T_{k}\left(u_{n}\right) d x \leq \frac{k}{m} \int_{\left\{m \leq\left|u_{n}\right| \leq 2 m\right\}} a\left(x, u_{n}, \nabla u_{n}\right) \nabla u_{n} d x
$$

inequality (3.35) becomes

$$
\begin{aligned}
& \int_{\Omega} a\left(x, T_{k}\left(u_{n}\right), \nabla T_{k}\left(u_{n}\right)\right) \nabla T_{k}\left(u_{n}\right)\left(1-h_{m}\left(u_{n}\right)\right) d x \\
\leq & \int_{\Omega} f_{n}\left(1-h_{m}\left(u_{n}\right)\right) T_{k}\left(u_{n}\right) d x+\frac{k}{m} \int_{\left\{m \leq\left|u_{n}\right| \leq 2 m\right\}} a\left(x, u_{n}, \nabla u_{n}\right) \nabla u_{n} d x .
\end{aligned}
$$

By passing to the limsup over $n$, one has

$$
\begin{aligned}
& \limsup _{n \rightarrow \infty} \int_{\Omega} a\left(x, T_{k}\left(u_{n}\right), \nabla T_{k}\left(u_{n}\right)\right) \nabla T_{k}\left(u_{n}\right)\left(1-h_{m}\left(u_{n}\right)\right) d x \\
& \quad \leq \int_{\Omega} f\left(1-h_{m}(u)\right) T_{k}(u) d x+\limsup _{n \rightarrow \infty} \frac{k}{m} \int_{\left\{m \leq\left|u_{n}\right| \leq 2 m\right\}} a\left(x, u_{n}, \nabla u_{n}\right) \nabla u_{n} d x .
\end{aligned}
$$

Passing again to the limsup, but now over $m$, we obtain

$$
\begin{array}{r}
\limsup _{m \rightarrow \infty} \limsup _{n \rightarrow \infty} \int_{\Omega} a\left(x, T_{k}\left(u_{n}\right), \nabla T_{k}\left(u_{n}\right)\right) \nabla T_{k}\left(u_{n}\right)\left(1-h_{m}\left(u_{n}\right)\right) d x \\
\leq \limsup _{m \rightarrow \infty} \limsup _{n \rightarrow \infty} \frac{k}{m} \int_{\left\{m \leq\left|u_{n}\right| \leq 2 m\right\}} a\left(x, u_{n}, \nabla u_{n}\right) \nabla u_{n} d x .
\end{array}
$$

On the other hand, since

$$
\frac{1}{m} \int_{\left\{m \leq\left|u_{n}\right| \leq 2 m\right\}} a\left(x, u_{n}, \nabla u_{n}\right) \nabla u_{n} d x \leq \int_{\left\{\left|u_{n}\right| \geq m\right\}}\left|f_{n}\right| d x
$$


one easily has

$$
\limsup _{m \rightarrow \infty} \limsup _{n \rightarrow \infty} \frac{1}{m} \int_{\left\{m \leq\left|u_{n}\right| \leq 2 m\right\}} a\left(x, u_{n}, \nabla u_{n}\right) \nabla u_{n} d x=0 .
$$

Hence, from (3.37) we deduce that

(3.38) $\limsup _{m \rightarrow \infty} \limsup _{n \rightarrow \infty} \int_{\Omega} a\left(x, T_{k}\left(u_{n}\right), \nabla T_{k}\left(u_{n}\right)\right) \nabla T_{k}\left(u_{n}\right)\left(1-h_{m}\left(u_{n}\right)\right) d x=0$.

Now, write

$$
\begin{aligned}
a\left(x, T_{k}\left(u_{n}\right), \nabla T_{k}\left(u_{n}\right)\right) & \nabla T_{k}\left(u_{n}\right) \\
= & a\left(x, T_{k}\left(u_{n}\right), \nabla T_{k}\left(u_{n}\right)\right) \nabla T_{k}\left(u_{n}\right) h_{m}\left(u_{n}\right) \\
& +a\left(x, T_{k}\left(u_{n}\right), \nabla T_{k}\left(u_{n}\right)\right) \nabla T_{k}\left(u_{n}\right)\left(1-h_{m}\left(u_{n}\right)\right),
\end{aligned}
$$

which gives, by (3.34) and (3.38),

$$
\begin{aligned}
\limsup _{m \rightarrow \infty} \limsup _{n \rightarrow \infty} \int_{\Omega} a\left(x, T_{k}\left(u_{n}\right), \nabla T_{k}\left(u_{n}\right)\right) \nabla T_{k}\left(u_{n}\right) d x & \\
& \leq \int_{\Omega} a\left(x, T_{k}(u), \nabla T_{k}(u)\right) \nabla T_{k}(u) d x
\end{aligned}
$$

and so

$$
\begin{aligned}
& \limsup _{n \rightarrow \infty} \int_{\Omega} a\left(x, T_{k}\left(u_{n}\right), \nabla T_{k}\left(u_{n}\right)\right) \nabla T_{k}\left(u_{n}\right) d x \\
& \quad \leq \int_{\Omega} a\left(x, T_{k}(u), \nabla T_{k}(u)\right) \nabla T_{k}(u) d x .
\end{aligned}
$$

On the other hand, thanks to Fatou's lemma, we have

$$
\begin{aligned}
\int_{\Omega} a\left(x, T_{k}(u), \nabla T_{k}(u)\right) & \nabla T_{k}(u) d x \\
& \leq \liminf _{n \rightarrow \infty} \int_{\Omega} a\left(x, T_{k}\left(u_{n}\right), \nabla T_{k}\left(u_{n}\right)\right) \nabla T_{k}\left(u_{n}\right) d x .
\end{aligned}
$$

Consequently, in view of (3.39), we obtain

$$
\begin{aligned}
\lim _{n \rightarrow \infty} \int_{\Omega} a\left(x, T_{k}\left(u_{n}\right), \nabla T_{k}\left(u_{n}\right)\right) & \nabla T_{k}\left(u_{n}\right) d x \\
& =\int_{\Omega} a\left(x, T_{k}(u), \nabla T_{k}(u)\right) \nabla T_{k}(u) d x .
\end{aligned}
$$

Thanks to (3.28) we have

$$
M\left(\left|\nabla T_{k}\left(u_{n}\right)\right| / \mu\right) \leq K^{\prime}+K M\left(\left|\nabla T_{k}\left(u_{n}\right)\right| / \lambda\right)
$$

and then by using (3.40), one obtains, by Vitali's theorem,

$$
M\left(\left|\nabla T_{k}\left(u_{n}\right)\right| / \mu\right) \rightarrow M\left(\left|\nabla T_{k}(u)\right| / \mu\right) \quad \text { in } L^{1}(\Omega) .
$$


SteP 4: Passage to the limit. By using $T_{k}\left(u_{n}-v\right)$ as test function in (3.8), with $v \in W_{0}^{1} L_{M}(\Omega) \cap L^{\infty}(\Omega)$, we get

$$
\begin{aligned}
& \int_{\Omega} a\left(x, T_{k+\|v\|_{\infty}}\left(u_{n}\right), \nabla T_{k+\|v\|_{\infty}}\left(u_{n}\right)\right) \nabla T_{k}\left(u_{n}-v\right) d x \\
& \quad+\int_{\Omega} g\left(x, u_{n}, \nabla u_{n}\right) T_{k}\left(u_{n}-v\right) d x=\int_{\Omega} f_{n} T_{k}\left(u_{n}-v\right) d x .
\end{aligned}
$$

By Fatou's lemma and the fact that

$$
a\left(x, T_{k+\|v\|_{\infty}}\left(u_{n}\right), \nabla T_{k+\|v\|_{\infty}}\left(u_{n}\right)\right) \rightarrow a\left(x, T_{k+\|v\|_{\infty}}(u), \nabla T_{k+\|v\|_{\infty}}(u)\right)
$$

weakly in $\left(L_{\bar{M}}(\Omega)\right)^{N}$ for $\sigma\left(\prod L_{\bar{M}}, \prod E_{M}\right)$ one easily sees that

$$
\begin{aligned}
\int_{\Omega} a(x, & \left.T_{k+\|v\|_{\infty}}(u), \nabla T_{k+\|v\|_{\infty}}(u)\right) \nabla T_{k}(u-v) d x \\
& \leq \liminf _{n \rightarrow \infty} \int_{\Omega} a\left(x, T_{k+\|v\|_{\infty}}\left(u_{n}\right), \nabla T_{k+\|v\|_{\infty}}\left(u_{n}\right)\right) \nabla T_{k}\left(u_{n}-v\right) d x
\end{aligned}
$$

Our next purpose is to prove that

$$
g\left(x, u_{n}, \nabla u_{n}\right) \rightarrow g(x, u, \nabla u) \quad \text { strongly in } L^{1}(\Omega) .
$$

In virtue of Vitali's theorem, it is sufficient to prove that $g\left(x, u_{n}, \nabla u_{n}\right)$ is equiintegrable in $L^{1}(\Omega)$. On the one hand, by taking $T_{1}\left(u_{n}-T_{l}\left(u_{n}\right)\right)$ as test function in (3.8), we obtain

$$
\int_{\left\{\left|u_{n}\right|>l+1\right\}}\left|g\left(x, u_{n}, \nabla u_{n}\right)\right| d x \leq \int_{\left\{\left|u_{n}\right|>l\right\}}\left|f_{n}\right| d x .
$$

Let $\varepsilon>0$. Then there exists $l(\varepsilon) \geq 1$ such that

$$
\int_{\left\{\left|u_{n}\right|>l(\varepsilon)\right\}}\left|g\left(x, u_{n}, \nabla u_{n}\right)\right| d x<\varepsilon / 2 .
$$

For any measurable subset $E \subset \Omega$, we have

$$
\begin{aligned}
\int_{E}\left|g\left(x, u_{n}, \nabla u_{n}\right)\right| d x \leq & \int_{E} b(l(\varepsilon))\left(c(x)+M\left(\left|\nabla T_{l(\varepsilon)}\left(u_{n}\right)\right| / \mu\right)\right) d x \\
& +\int_{\left\{\left|u_{n}\right|>l(\varepsilon)\right\}}\left|g\left(x, u_{n}, \nabla u_{n}\right)\right| d x .
\end{aligned}
$$

In view of (3.41) there exists $\eta(\varepsilon)>0$ such that

$$
\int_{E} b(l(\varepsilon))\left(c(x)+M\left(\left|\nabla T_{l(\varepsilon)}\left(u_{n}\right)\right| / \mu\right)\right) d x<\varepsilon / 2
$$

for all $E$ such that $|E|<\eta(\varepsilon)$.

Finally, by combining (3.44) and (3.45) one easily has

$$
\int_{E}\left|g\left(x, u_{n}, \nabla u_{n}\right)\right| d x<\varepsilon \quad \text { for all } E \text { such that }|E|<\eta(\varepsilon),
$$

which allows us, by using (3.43), to pass to the limit in (3.42).

This completes the proof. 
REMARK 3.3. We obtain the same result of our theorem if we replace (3.1) by the general growth condition

$$
|a(x, s, \zeta)| \leq \bar{b}(s)\left(\bar{h}(x)+\bar{M}^{-1} M(k|\zeta|)\right)
$$

where $k \geq 0, \bar{h} \in E_{\bar{M}}(\Omega)$ and $\bar{b}: \mathbb{R}_{+} \rightarrow \mathbb{R}$ is a continuous nondecreasing function. Indeed, we consider the following approximate problems:

$$
\left\{\begin{array}{l}
-\operatorname{div}\left(a\left(x, T_{n}\left(u_{n}\right), \nabla u_{n}\right)\right)+g\left(x, u_{n}, \nabla u_{n}\right)=f_{n} \quad \text { in } \mathcal{D}^{\prime}(\Omega), \\
u_{n} \in W_{0}^{1} L_{M}(\Omega), \quad g\left(x, u_{n}, \nabla u_{n}\right) \in L^{1}(\Omega), \quad g\left(x, u_{n}, \nabla u_{n}\right) u_{n} \in L^{1}(\Omega),
\end{array}\right.
$$

and we end the proof by using the same last steps.

For some results obtained in the $L^{p}$ case under the assumption (3.46), we refer to [19] and [20].

\section{References}

[1] R. Adams, Sobolev Spaces, Academic Press, New York, 1975.

[2] P. Bénilan, L. Boccardo, T. Gallouët, R. Gariepy, M. Pierre and J. L. Vázquez, An $L^{1}$-theory of existence and uniqueness of nonlinear elliptic equations, Ann. Scuola Norm. Sup. Pisa 22 (1995), 240-273.

[3] A. Benkirane, Approximation de type Hedberg dans les espaces $W^{m} L \log L(\Omega)$ et applications, Ann. Fac. Sci. Toulouse 11 (1990), 67-78.

[4] A. Benkirane and A. Elmahi, Almost everywhere convergence of the gradients of solutions to elliptic equations in Orlicz spaces and application, Nonlinear Anal. 28 (1997), 1769-1784.

[5] —, - An existence theorem for a strongly nonlinear elliptic problem in Orlicz spaces, ibid. 36 (1999), 11-24.

[6] - - - A strongly nonlinear elliptic equation having natural growth terms and $L^{1}$ data, ibid. 39 (2000), 403-411.

[7] A. Benkirane and J.-P. Gossez, An approximation theorem for higher order OrliczSobolev spaces, Studia Math. 92 (1989), 231-255.

[8] A. Bensoussan, L. Boccardo and F. Murat, On a nonlinear partial differential equation having natural growth terms and unbounded solution, Ann. Inst. H. Poincaré 5 (1988), 347-364.

[9] L. Boccardo, Some nonlinear Dirichlet problems in $L^{1}$ involving lower order terms in divergence form, in: Progress in Elliptic and Parabolic Partial Differential Equations (Capri, 1994), Pitman Res. Notes Math. Ser. 350, Longman, Harlow, 1996, 43-57.

[10] L. Boccardo and T. Gallouët, Nonlinear elliptic and parabolic equations involving measure data, J. Funct. Anal. 87 (1989), 149-169.

[11] - - - Strongly nonlinear elliptic equations having natural growth and $L^{1}$ data, Nonlinear Anal. 19 (1992), 573-578.

[12] L. Boccardo, T. Gallouët and F. Murat, A unified presentation of two existence results for problems with natural growth, in: Progress in Partial Differential Equations: the Metz Surveys 2, M. Chipot (ed.), Pitman Res. Notes Math. Ser. 296, Longman, 1993, 127-137.

[13] L. Boccardo, T. Gallouët and L. Orsina, Existence and nonexistence of solutions for some nonlinear elliptic equations, J. Anal. Math. 73 (1997), 203-223.

[14] L. Boccardo, F. Murat and J.-P. Puel, Existence of bounded solutions for nonlinear elliptic unilateral problems, Ann. Mat. Pura Appl. 152 (1988), 183-196. 
[15] J.-P. Gossez, Nonlinear elliptic boundary value problems for equations with rapidly (or slowly) increasing coefficients, Trans. Amer. Math. Soc. 190 (1974), 163-205.

[16] - Some approximation properties in Orlicz-Sobolev spaces, Studia Math. 74 (1982), $17-24$.

[17] - A strongly nonlinear elliptic problem in Orlicz-Sobolev spaces, in: Proc. Sympos. Pure Math. 45, Amer. Math. Soc., 1986, 455-462.

[18] J.-P. Gossez and V. Mustonen, Variational inequalities in Orlicz-Sobolev spaces, Nonlinear Anal. 11 (1987), 379-392.

[19] C. Leone and A. Porretta, Entropy solutions for nonlinear elliptic equations in $L^{1}$, Nonlinear Anal. 32 (1998), 325-334.

[20] A. Porretta, Some remarks on the regularity of solutions for a class of elliptic equations with measure data, Houston J. Math. 26 (2000), 183-213.

[21] - Existence for elliptic equations in $L^{1}$ having lower order terms with natural growth, Portugal. Math. 57 (2000), 179-190.

Département de Mathématiques et Informatique

C.P.R. de Fès

Faculté des Sciences Dhar-Mahraz

B.P. 49, Fès, Maroc

B.P. 1796 Atlas Fès, Maroc

E-mail: abdelmoujib@iam.net.ma

meskinedriss@hotmail.com 